\title{
Gestión pública para erradicar la pobreza: Las soluciones existen
}

\author{
Azcueta, Michel*
}

\section{Resumen}

Este ensayo tiene como propósito analizar la evolución de la pobreza e inequidad en el mundo, su relación con el modelo de desarrollo impulsado durante la globalización y evaluar las respuestas que los gobiernos han intentado para enfrentarla. Se establece la distinción entre programas de emergencia y programas de desarrollo; se precisan los rasgos fundamentales de experiencias concretas exitosas, y las propuestas para erradicar la pobreza. Se concluye con la recomendación de un conjunto de estrategias para el éxito de las propuestas para erradicar la pobreza.

Palabras clave: Gestión pública, gobiernos locales, pobreza.

\section{Public Management to Eradicate Poverty: The Solutions Exist}

\begin{abstract}
The purpose of this essay is to analyze the evolution of poverty and inequality in the world, to relate them with development models promoted during globalization, and to evaluate the responses that governments have attempted in order to confront it. A distinction is established between emergency programs and development programs; fundamental characteristics of concrete and successful experience and proposals to eradicate poverty. The essay concludes with a recommendation as to a set of strategies for success in poroposals to eliminate poverty.
\end{abstract}

Key words Public management, local government, poverty.

Recibido: 02-02-26 . Aceptado: 02-07-08

Regidor Concejo Municipal de Lima. Ex - Alcalde de Villa El Salvador (Perú). 


\section{Introducción}

Son ya varias las décadas que tanto la Organización de Naciones Unidas como los organismos internacionales y los propios gobiernos han dedicado grandes esfuerzos al "desarrollo", y a la lucha contra la pobreza probando prácticamente de todo, con resultados mínimos en comparación con las demandas existentes de la población pobre tanto en los países del Sur, como en los países de alto nivel de desarrollo.

Se han ido focalizando aspectos que se iban poniendo de moda de acuerdo a los años, a los enfoques de especialistas muy lúcidos y convencidos y de acuerdo, también, a intereses políticos de corto plazo. De ahí tenemos varias experiencias de programas de desarrollo que se han ido centrando en el campo, en las creas urbanas, en la salud, en las mujeres o "política de género", en la infancia, a veces, en la alfabetización y la educación básica...., también se han ido probando diferentes métodos desde el propio Estado, Ministerios, organismos asistencialistas, ONGs, cooperación internacional directa e indirecta, así como diferentes sistemas de control y evaluación de los múltiples proyectos y programas de lucha contra la pobreza.

Los resultados, salvo algunas excepciones muy localizadas en uno $u$ otro país, no son muy alentadores: miles de millones de dólares invertidos en estos programas para que se mantenga el mismo número de pobres, cuantitativamente hablando, y el mismo número de ciudadanos en pobreza extrema en la mayoría de nuestros países en todos los continentes y en el conjunto de América
Latina, África y Asia, aumentando los llamados "bolsones" de pobreza en los países desarrollados.

Al entrar al siglo XXI, conviene evaluar profundamente dichos programas en sus objetivos, métodos y resultados, resaltando las experiencias positivas, con éxitos comprobados y, a partir de esta evaluación, asumir con valentía los cambios propiciando una voluntad política y ciudadana para enfrentar la pobreza y avanzar en el desarrollo integral de los pueblos.

\section{Algunas constataciones previas}

Aunque siempre se han producido numerosas evaluaciones y discusiones sobre las causas de la pobreza en el mundo, que incluyen análisis históricos, económicos, culturales y sociales, hay que profundizar aún más sobre estas causas, a la luz de los fracasos que se han dado y se siguen dando en los programas de lucha contra la pobreza, acercándonos más aún a las causas próximas que debemos y podemos atacar para llegar a las causas estructurales que todos conocemos y que siguen siendo la base de las situaciones de pobreza. Considero que, en muchas ocasiones, las causas estructurales, que son ciertas y reales, las aceptamos todos -organismos internacionales, gobiernos y ciudadanía en general - $y$, por esa misma razón, las mantenemos en una especie de "limbo", de manera que su conocimiento y constatación ya no movilizan ni animan a la acción concreta, dejando de ser, en muchas ocasiones, un auténtico motor de promoción del desarrollo. Peor aún, desde algunos gobiernos, las causas estructurales se utilizan como 
un escudo para salvar, según ellos, responsabilidades, ya que "si siempre ha sido así, poco se puede hacer".

Los resultados en las políticas económicas y sociales de parte de los organismos internacionales son tan claros que nadie los puede negar.

"Por un lado, en la dimensión distributiva del desarrollo económico, América Latina ocupa una posición desfavorable: en forma persistente, la región ha tenido índices muy altos de concentración del ingreso, superiores a las de cualquier otra región. En los países latinoamericanos una cuarta parte del ingreso nacional es percibida por solo el $5 \%$ de la población y un $40 \%$ por el $10 \%$ más rico. En contraste, en los países del sudeste asiático el $5 \%$ más rico percibe el $16 \%$ del ingreso promedio y en los países desarrollados, el 13\%" (BID - 1999).

Por eso, sin dejar de lado lo relacionado con este tema, conviene asumir los cambios efectuados en el mundo, en las regiones y en cada uno de nuestros paí- ses, para atender las demandas concretas en un contexto diferente, donde los intereses de las personas consideradas pobres y pobres extremos son muy específicos para, a partir de ellos avanzar hacia un desarrollo integral.

Junto con este nuevo análisis, también se deben evaluar los modelos de desarrollo implícitos en los programas y proyectos de lucha contra la pobreza. $\mathrm{Si}$, en la práctica, han fracasado las consideraciones fundamentales, también han fracasado los modelos; según podemos constatar, lo que se ha logrado es una modernización real de sectores minoritarios en cada uno de nuestros países y una auténtica marginación de la mayoría pobre, sobre todo en los países del Sur (Ver Gráfico 1).

De nuevo las cifras oficiales nos demuestran los resultados de este modelo de desarrollo. Según el último informe de Naciones Unidas (PNUD) "en los últimos 30 años, la diferencia de ingresos "per-capita" entre los países pobres y ri-

\section{Gráfico 1 \\ Dónde viven los pobres del mundo en desarrollo}

Distribución de la población que vive con menos de US\$1 diario, 1998 (1.200 millones)

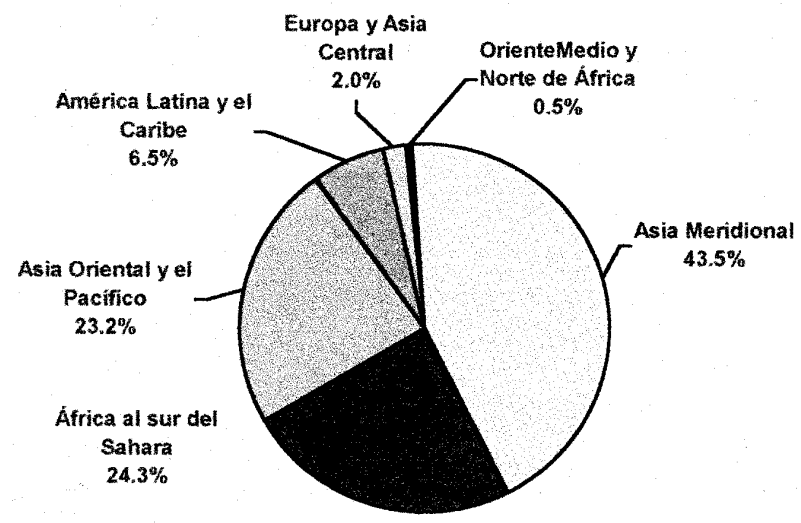

Fuente: Banco Mundial, 2000b. 
cos ha pasado de 19 a 30. Y esta diferencia se ha agudizado al interior mismo de los países ricos. En EE.UU ha pasado de 1.9 a 60 . El ingreso de los ricos que conforman el $1 \%$ de la población, se ha multiplicado por 100, mientras el ingreso de la población pobre que representa el $20 \%$ se ha mantenido igual o ha empeorado" (Informe PNUD-2000).

Pareciera que el modelo de desarrollo elegido en la última década tiene como fin exclusivo enlazar ese sector minoritario moderno con la llamada "globalización", de manera que se fortalece la paradoja de una presencia efectiva de dicho sector minoritario en las redes internacionales y una ausencia real de las mayorías nacionales que quedan al margen de ese proceso mundial.

Esta globalización económica, financiera y de imagen, no termina de solucionar los problemas concretos de millones de personas ni logra adecuar las demandas, tan inmediatas y profundas, de ciudadanos/nos de todas las regiones y países del planeta.

Es por esta razón que la "globalización", a pesar de las tensiones con que es observada respecto al desarrollo de las localidades, paradójicamente incentivo una sensibilización hacia lo local, que hay que tener en cuenta aunque siempre fomentando visiones y estrategias innovadoras de desarrollo que no fueron probadas anteriormente.

Es la propia "globalización" lo que nos lleva al redescubrimiento de lo local, haciendo cobrar un valor especial a las discusiones y valorizaciones sobre identidad, participación y desarrollo local, haciendo de éste un referente de un quehacer social y político de transformación que esta teniendo gran influencia en los inicios del nuevo siglo. Después de 20 años ensalzando o criticando la "globalización", cualquiera sea la posición, hacen de las estrategias de desarrollo local constituyen un objetivo político común de diferenciación, de configuración de un poder compensador de los procesos y agentes que actualmente hegemonizan a la humanidad.

Los municipios y las instituciones municipales son los que mejor expresan estas posibilidades en todos los países del mundo. Mientras el gobierno central garantizan la igualdad de derechos de los ciudadanos/nas, el gobierno local garantiza el derecho a las diferencias.

Por lo tanto, en los nuevos programas y proyectos para reducir la pobreza en América Latina, el Caribe, Africa y Asia debemos considerar, también, esta nueva dimensión del desarrollo para hacer posible que las grandes mayorías participen activamente en la construcción de las relaciones internacionales propias del nuevo Milenio. Atender las demandas concretas de la población pobre, pero con una nueva dimensión, sabiendo unir desarrollo y democracia.

Otro de los aspectos que se suele dejar de lado tanto en la constatación como en las políticas de lucha contra la pobreza es la imagen de los pobres y de la misma pobreza que se transmite a través de los diferentes medios de comunicación, especialmente por la televisión, a nivel mundial $y$, muy especialmente, en los países desarrollados.

Aunque, de forma individual podemos y solemos tener nuestros propios criterios de evaluación, desde el punto de vista social y cultural masivo, se sigue re- 
lacionando, en los medios, la pobreza con actitudes individuales negativas, propias de sectores minoritarios de la sociedad (en los países desarrollados) o con poblaciones culturalmente "atrasadas" y sin iniciativa para combatir su situación. Generalmente, no se presentan las noticias sobre lo pobreza de manera profunda e integral sino como hechos aislados producto de una situación coyuntural que, como suele ocurrir en la presentación televisiva de hambrunas, catástrofes o epidemias en el Tercer Mundo, que nos mueven a cierta compasión pero casi nunca inducen a una reflexión masiva sobre las causas de la pobreza en el mundo y su relación directa con decisiones que se toman a nivel de gobierno y a nivel internacional.

Es cierto que se dan excepciones, como lo demuestran muchos periodistas y comentaristas en el mundo entero. Por ejemplo, en los Encuentros "Sur y Medios de Comunicación" realizados en Pamplona - España, en 1997, se concluye."En la era de las comunicaciones, del desarrollo de nuevas tecnologías, se está produciendo una revolución en el concepto clásico de información y debemos estar alerta ante algunas cualidades del nuevo modelo: la información es unidireccional la información es universal pero uniforme. Estas características de la información actual inciden de manera especialmente negativa cuando se aplican a la realidad de los pueblos empobrecidos del planeta y económicamente irrelevantes para el mercado mundial" (Médicos Mundi, 1999).

Este control de la imagen en relación con la lucha contra la pobreza, se refleja, también, en la información sobre los programas de cooperación en el Tercer
Mundo. El Comité de enlace de las ONG nos describe algunos ejemplos:

"Son también muy comunes las imágenes que transmiten la idea de que médicos, maestros, ingenieros, y en general, todo el personal verdaderamente capacitado, es siempre blanco y occidental, lo que refleja que todavía no se termina de poner en manos de las contrapartes nativas la administración y ejecución de los proyectos, tal y como sería deseable. Tienen, así mismo, connotaciones paternalistas, las fotografías que presentan al personaje occidental, sano, fuerte y estupendo, agarrando la manita de un niño esquelético; una imagen que vuelve a transmitir la debilidad, la dependencia, la infancia del Tercer Mundo necesitada de nuestra ayuda. Lo son, por último, algunos pies de foto en los que se lee, por ejemplo, "Pedro Pérez, director de tal Agencia, abraza a un niño etíope" donde, al parecer, el director de la ONG merece ser descrito con nombre, apellido profesión, mientras el niño es sólo un representante abstracto de la infancia etíope desvalida.

Hay, por último, algunas manifestaciones muy sutiles o indirectas de prejuicio o discriminación racial" (citado en Médicos Mundi, 1999).

Una última constatación, que considero útil y necesaria es aceptar la distinción entre "programas de emergencia" y "programas de desarrollo".

Lamentablemente, los "programas de emergencia" ante catástrofes naturales, epidemias, hambrunas, situaciones graves imprevistas, siguen y seguirán siendo necesarios en los países del Tercer Mundo, dada nuestra realidad geográfica, económica y social. Con la expe- 
riencia adquirida, deben mantenerse programas y presupuestos para la acción preventiva, para la información y educación de la población de zonas afectadas por terremotos, ciclones, erupciones volcánicas, epidemias, sequías e inundaciones, población que definitivamente está dentro de los sectores más pobres de nuestras sociedades lo cual hace que las catástrofes naturales en nuestros países sean también factor de empobrecimiento $y$ de miseria.

A pesar de ello, mucho se puede hacer para no repetir errores ni de parte de la propia población ni de parte de los gobiernos y organismos internacionales que movilizan recursos ante catástrofes, logrando ayudas de emergencia, pero no concluyen en otro tipo de acciones con objetivos de corto y mediano plazo.

De ahí la necesidad e importancia de la distinción entre estos "programas de emergencia" - orientados en su mayoría a la población pobre - y los "programas de desarrollo integral" que se encaminan a soluciones definitivas. Precisamente sobre este último aspecto debemos profundizar y avanzar.

\section{Las soluciones existen}

Ante tanto fracaso y por las propias evaluaciones objetivas de los programas de lucha contra la pobreza, se han ido analizando, tanto a nivel nacional como internacional, experiencias concretas que terminan en éxito como ejemplos que pudieran ser replicados en otras zonas y países. Más allá de que puede significar una nueva "moda" o simple justificación para dichos programas, el hecho concreto es que existen, en todos los continen- tes experiencias que señalan algunos caminos concretos por los cuales deberíamos avanzar en la solución al problema de la pobreza.

Podemos encontrar algunas de las más conocidas en las últimas publicaciones referentes al tema como, por ejemplo, los informes anuales sobre las mejores experiencias urbanas del programa "Habitat" de Naciones Unidas; las 17 experiencias recogidas por el Banco Mundial en "Our Dream: a World Free of Poverty" (Washington - 2000); las 32 experiencias de América Latina en el "Kit Ciudades y Medio Ambiente", de la Federación Mundial de Ciudades Unidas (París 2000); el estudio del grupo "Laboratoire du Futur" (París - 2000) sobre cinco acciones concretas de lucha contra la pobreza en Bangladesh (Grameen Bank), Estados Unidos (Time dollar), Holanda y Bélgica (Max Havelaor), Brasil (Presupuesto participativo de Porto Alegre) y Perú (Desarrollo integral de Villa El Salvador), experiencia esta última en la que participo directamente desde 1971 hasta el presente.

Se trata de acciones que se desarrollan a partir de un eje específico: desarrollo económico, social, educativo, etc., en ambientes urbanos o rurales, que guardan relación con temas considerados tradicionales (salud, alimentación, trabajo, producción, organización, etc.) pero que han ido incorporando otros relacionados con la pobreza en el mundo (inmigración, grupos minoritarios, comunicación, identidad nacional, pacificación, derechos humanos, globalización, etc.)

Son centenares y centenares de esfuerzos en todos los continentes y países (incluidos los países desarrollados y 
sus "bolsones" de pobreza) que, guardando las distancias y el sector social y poblacional específico en el que se desarrollan, mantienen ciertas coincidencias y similitudes que conviene recoger:

- son experiencias locales con dimensión nacional: parten de lo local pero no se quedan ahí, sino que construyen puentes de relación y coordinación con otros grupos y con otros sectores, a veces, absolutamente diferentes, con el fin de ampliar su campo de acción, aumentar su fuerza e influir en políticas nacionales e internacionales

- son experiencias que fortalecen la sociedad civil : en cada uno de los países, se diferencian de las acciones que llevan a cabo los gobiernos centrales, logrando un "empoderamiento" de los pobres para dirigir sus propios procesos de desarrollo, sobre todo cuando culminan en el fortalecimiento institucional

- Son experiencias de democracia interna: que tienen como objetivo fortalecer los procesos democráticos nacionales e internacionales, construyendo nuevos liderazgos comunales, municipales, políticos, económicos, resaltando los liderazgos de mujeres y de jóvenes.

- Son experiencias que inciden en proyectos integrales: como manera de atacar la situación de pobreza. Más allá del "eje" concreto elegido (productivo, educativo, social, cultural...) se enmarcan siempre en un proyecto integral de desarrollo, con diferentes acciones y actores coordinados entre sí para lograr objetivos de corto, mediano y largo plazo.
- Son experiencias que fortalecen la identidad del grupo: generando un orgullo propio entre sus integrantes y logrando un alto nivel de reconocimiento desde los sectores externos a la experiencia. Esta identidad propia contribuye al proceso de construcción de nuevas identidades nacionales en varios países de Asia, Africa y América Latina o al fortalecimiento de identidades en sectores minoritarios de países desarrollados.

- Son experiencias que modifican actitudes en los participantes: fortaleciendo la auto-confianza, aspecto importantísimo en la lucha contra la pobreza, ya que todo objetivo alcanzado se transforma en un éxito personal y colectivo, disminuyendo o alejando las frustraciones, una de las características de la cultura de la pobreza.

Es aprendiendo de estas experiencias positivas que se debe avanzar en la construcción de un sistema y unos modelos de desarrollo económico y social que conduzcan tanto a la inmediata reducción de la pobreza como a su futura eliminación definitiva.

\section{Propuestas para la acción}

a) Desarrollo local integral.

En base a las experiencias que han demostrado ciertos niveles de éxito en la elevación del nivel de vida de poblaciones consideradas pobres, proponemos dar más importancia a proyectos integrales a nivel local, a partir de núcleos generadores de experiencias productivas.

No se trata, simplemente, de mantener una situación donde la pobreza no siga avanzando. Se trata de generar ri- 
queza, iniciar o mejorar la producción a nivel local, para asegurar un sostenimiento básico en la familia y aumentar las posibilidades personales de mejorar, en un futuro próximo, la propia situación. De la pobreza en sí no puede salir la solución. Es necesario romper el círculo vicioso - la pobreza genera más pobreza - a través de lo productivo en base a las ventajas comparativas y recursos básicos de una comunidad local.

La mejora económica incide directa e inmediatamente en la mejora de los servicios elementales como la higiene, la salud, la educación y no necesariamente se produce la situación inversa. Hay que centrarse en lo económico-productivo. Nuestra experiencia personal de más de tres décadas en Villa El Salvador, un distrito popular de cerca de 400,000 habitantes en el área metropolitana de Lima - Perú, nos ha enseñado a valorar el trabajo productivo de hombres, mujeres y jóvenes como la mejor manera de salir de la pobreza extrema y comenzar a progresar.

b) Concertación.

Es cierto que, en todo lo que se refiere a la producción es necesario concertar entre los diferentes actores sociales. población organizada, empresarios, gobierno local, gobierno central y ONGs. La concertación por y para el desarrollo, es la base para reducir la pobreza y para fortalecer la democracia.

Proyectos productivos enmarcados en un plan de desarrollo integral a nivel local, de manera tal que, al ir elevando y mejorando la producción, se acercan los mercados, se abren nuevas y mejores relaciones con otras zonas, inclusive las más alejadas, y se va participando en un plan nacional de desarrollo, fortaleciendo la conciencia ciudadana y la consolidación de derechos y deberes. Precisamente, en mi opinión, es la conciencia de derechos y obligaciones la que demostrará que se está saliendo de la miseria y de la marginación absolutas.

c) Integración de objetivos sociales, económicos y políticos.

De ahí la importancia del componente democrático en todo plan de desarrollo integral. El auténtico reto está en saber unir democracia con eficacia, participación con resultados concretos, producción y empleo con justicia social.

La democracia debe ser un objetivo conscientemente asumido dentro de los programas de cooperación hablado, especialmente, de América Latina, África y Asia. La Comunidad Democrática, los organismos de la Organización de Naciones Unidas, debe saber transmitir los valores propios de la democracia en cada uno de los proyectos de cooperación y, consecuentemente, se debe encontrar la mejor manera y tos caminos convenientes para influir en la democratización de las sociedades a las cuales de destina la cooperación.

d) Fortalecimiento de gobiernos locales participativos.

En base a la experiencia personal, me atrevo a decir que uno de los caminos seguros es el fortalecimiento de los gobiernos locales. En las últimas dos décadas se han ido generalizando las elecciones libres en el ámbito municipal y, poco a poco, con tristes excepciones, han ido asumiendo nuevos roles y nuevas responsabilidades en diferentes ámbitos del desarrollo económico, social y cultural. Por eso considero importante tener en cuenta a los municipios y, junto con ellos, 
la participación de las organizaciones de base que forman la comunidad local, a la hora de definir los objetivos, programas y proyectos de cooperación internacional. El fortalecimiento de la institucionalidad democrática de la sociedad civil debe ser un elemento importante, $y$, a su vez, uno de los logros visibles de toda cooperación orientada a la erradicación de la pobreza en el mundo.

e) Énfasis en el desarrollo educativo y cultural.

En el contexto de un desarrollo integral y de ninguna manera aislados es que consideramos los importantes aspectos de la educación, la cultura y la información. Para ello, en las próximas décadas debemos producir cambios en todos los actores que participan en los tres procesos, cambios en los contenidos educativos y culturales, cambios en las metodologías, partiendo del respeto a los pobres tanto como personas individuales, así como a los grupos sociales que conforman.

La educación, en los países y zonas pobres subdesarrolladas, así como en los "bolsones" de pobreza del mundo desarrollado, debe insistir en la construcción y transmisión de valores y conocimientos que generen auto-estima y disminuyan las frustraciones. No se trata solamente de alfabetizar o de aumentar los niveles de escolaridad en dichos sectores sino, también y además de eso, ayudar a descubrir sus propias potencialidades internas, tanto individuales como grupales, para que se reconozcan y sean reconocidos como sujetos capaces de alcanzar niveles superiores de desarrollo $y$, a partir de ello, participar con aportes propios en el desarrollo. Para lograr este objetivo es fundamental introducir en los procesos educativos, la "pedagogía del éxito", aquella que se basa en objetivos alcanzados que estimulan la confianza en la posibilidad real de alcanzar objetivos más importantes. La "pedagogía del éxito" puede y debe ser aplicada en todos los niveles y sectores, entre los niños, los jóvenes y los adultos hombres y mujeres que viven, hoy por hoy, en la pobreza.

La cultura o las culturas tienen que asumir el reto de la universalización en las relaciones humanas. Siempre seguirá siendo manifestación de la actividad, intelectual y práctica, de los miembros que conforman una comunidad determinada; pero en el Siglo XXI, más que en otros periodos de la historia de la humanidad, se debe insistir, por un lado, en el respeto a las culturas propias y, por otro, en los aportes a la cultura universal. Esto es absolutamente válido cuando hablamos de la erradicación de la pobreza en el mundo. Consciente o inconscientemente, a través de los modelos de desarrollo y a través de numerosos programas nacionales e internacionales, se transmiten ( $y$, a veces, se imponen....) otros valores y otros conocimientos que configuran otra cultura, despreciando en la práctica, la cultura propia de los sectores y naciones que se pretende apoyar, cerrando las puertas a sus propios aportes culturales, tan positivos como los de las demás culturas. No se puede combatir la pobreza destruyendo la cultura y las creaciones de aquellos que circunstancialmente, viven en ella.

f) Facilitar el acceso a tecnologías de información y comunicación.

La información es fundamental, tanto al interior de los grupos y socieda- 
des como en los ámbitos regionales y mundiales. De ahí la importancia de construir y mantener los accesos a la información y, lógicamente, a los medios y tecnologías de la comunicación. Cuando nos referimos a las estrategias de lucha contra la pobreza, lo hacemos con una visión integral de manera que debemos considerar un manejo adecuado de la información y de los medios de comunicación al servicio, también, de los objetivos de desarrollo, de las dimensiones culturales, con sus diferencias, y por la construcción de unas relaciones universales distintas a las existentes.

La tecnología comunicacional tiene la ventaja de su rápida expansión y de su abaratamiento creciente que la hace asequible cada vez más a sectores más amplios de la sociedad, incluidos los más pobres. Este es un aspecto muy positivo. El aspecto negativo o difícil es el de los contenidos que se transmiten así como la imagen que imponen al mundo sobre la realidad de los países pobres y de las minorías pobres, por lo que hay que trabajar en los dos niveles: haciendo que dichos sectores se apropien de diferentes mecanismos de información y comunicación y, por otra parte, lograr que, a nivel mundial, se generen nuevos canales y nuevos contenidos relacionados con un verdadero modelo de desarrollo económico, social y cultural.

g) Autosostenibilidad y cooperación internacional.

Junto con la concertación, recordemos que las experiencias también nos han enseñado que la cooperación internacional debe entenderse como un complemento a las acciones de cada grupo humano o de cada comunidad local. Generalmente, proyectos que se inician con financiamiento exterior terminan cuando se va ese apoyo exterior. Definitivamente es mucho más útil -hablando de desarrollo y no de emergencia- apoyar proyectos económico-productivos que han sido iniciados por la propia población pobre, con un núcleo estable y con objetivos claros (que pueden ser mejorados) para asegurar la continuidad del proyecto una vez terminada la cooperación internacional. De igual manera en lo que se refiere a proyectos y programas culturales y científicas. Una buena evaluación de los proyectos de la cooperación internacional tanto de los que terminan en éxito como de los que culminan en fracaso, ayudaría realmente a definir mejor las estrategias y métodos de lucha contra la pobreza y el fortalecimiento de la democracia.

\section{Recomendaciones}

El éxito de los programas tendientes a superar la pobreza exige:

Clara voluntad política: de parte de todos y, de manera especial de aquellos que tenemos algún tipo de responsabilidad pública a nivel local, nacional e internacional. Mientras no estemos convencidos no sólo de la necesidad de erradicar la pobreza en el incipiente Siglo XXI sino, también, de que este objetivo es realmente alcanzable con el aporte de todos, difícilmente vamos a poder avanzar hacia unas relaciones económicas y sociales más democráticas y justas. La voluntad política supone proponerse, de manera consciente y utilizando todos los recursos técnicos, humanos y económi- 
cos posibles, la erradicación de la pobreza, como gran objetivo mundial.

Relacionar la inversión nacional e internacional con el desarrollo local: teniendo en cuenta que prácticamente todas las experiencias con éxito en la lucha contra la pobreza se inician en espacios locales o grupos sociales concretos y, desde ahí, expanden su influencia a niveles más amplios. No se trata de despreciar acciones en el campo de la macroeconomía, absolutamente necesarias, sino asegurar que estas decisiones que se toman desde los gobiernos centrales y desde los organismos internacionales lleguen realmente a las poblaciones y grupos pobres de la sociedad. Esta es, a la vez, una demanda y una lección de la evaluación de los programas de lucha contra la pobreza.

\section{Respeto y desarrollo de concien-}

cia ciudadana: a partir del conocimiento y difusión de los deberes y derechos de cada uno de los habitantes de nuestro único planeta. Conciencia ciudadana quiere decir transformarnos todos, más allá de nuestra ubicación social y geográ fica, en sujetos activos de la sociedad civil, capaces de participar en la construcción de un mundo nuevo, sin exclusiones ni pobrezas de ningún tipo. Es a partir de este compromiso individual que, sumándose al compromiso de los demás, se irá fortaleciendo una conciencia colectiva, fundamento real de una "sociedad civil universal" cohesionada y con relaciones de igualdad.

Fomentar experiencias de éxito: una de las características de la historia de las sociedades y grupos pobres, mirada en su conjunto, es que se siente un balan- ce negativo, con más fracasos que éxitos en la tarea colectiva, lógicamente con las naturales excepciones. Demasiados intentos que acaban en frustraciones, demasiadas cosas que quedan a medio camino, demasiada mediocridad, demasiadas buenas intenciones, mucho engaño y mucha mentira... Todo ello influye en los sectores pobres y genera un sentimiento de frustración e impotencia que bloquean posibilidades ciertas de superar la pobreza. Por eso, debemos fomentar experiencias que culminen en éxitos concretos, en objetivos alcanzados que permiten y animan plantearse objetivos superiores.

Valorar la imaginación y creatividad: después de todo lo que hemos vivido en estas décadas y de los fracasos demostrados en la erradicación de la pobreza no sólo no hay que temer a las ideas y prácticas innovadoras sino, por el contrario, atreverse a aceptar la imaginación y creatividad de todos aquellos que desean aportar alguna solución. Fijarse más en las ideas, en las prácticas y propuestas que vienen de los jóvenes, de los intelectuales, de los artistas que, generalmente, suelen tener una sensibilidad especial para manifestar sus puntos de vista y sus preocupaciones sobre temas tan importantes como la desigualdad, lo solidaridad, los derechos humanos y sobre los problemas concretos de personas individuales que pueden transformarse en símbolos de las actividades y logros en la lucha contra la pobreza.

Fomentar la participación de las mujeres y jóvenes, quienes cargan el peso mayor de las consecuencias de la pobreza en todos los países; en la medida de lo posible, participación desde y con 
sus propias organizaciones, que son las que garantizan la continuidad de los programas y su expansión. Por un lado, las mujeres, especialmente en las zonas pobres de África, Asia y América Latina, tienen un circulo de relaciones y una actividad social, familiar y pública que, en muchos casos, es decisiva para formar actitudes ante la vida y ante la sociedad, por lo cual fomentar liderazgos entre las mujeres en el marco de proyectos integrales de lucha contra la pobreza, tiene repercusiones altamente positivas. Más aún, está demostrado que las organizaciones de mujeres tiene mayor estabilidad y continuidad que otro tipo de organizaciones sociales, siendo auténticos puentes para una participación consciente.

Los jóvenes, por su parte, tienen muy internalizadas las ideas de progreso y superación del estado de pobreza. Es cierto que las frustraciones y la falta de continuidad de las organizaciones juveniles, natural por la propia edad, representan una dificultad, pero también es cierto que muchos de los lideres juveniles se transforman, después, en dirigentes sociales y políticos en diferentes ámbitos (local, municipal, empresarial o al interior de los partidos y movimientos políticos), con influencia y poder representativo, por lo cual es importante que en los programas integrales de lucha contra la pobreza, se consideren líneas de acción específicas con la juventud.

Promover programas de información y comunicación en la línea que hemos señalado anteriormente, al interior del propio grupo social con el objetivo de cohesionarlo y fortalecer su identidad, $y$ hacia el exterior creando mecanismos de intercambio de experiencias, de conocimiento de otras realidades, y para, fundamentalmente, cambiar de raíz la imagen de la pobreza y de los pobres transmitida por la mayoría de los medios de comunicación tanto en el Norte como en el Sur. En este campo, los programas deben incluir un trabajo específico con periodistas, comunicadores, especialistas y empresarios dueños de los medios de comunicación, tanto para generar una nueva conciencia sobre el problema de la pobreza como sobre los contenidos que se transmiten sobre este tema.

Así mismo, es necesario apoyar las numerosas experiencias de radio y televisión comunitarias que son propiedad de comunidades locales y de grupos sociales minoritarios, porque representan una cuota concreta de poder en el campo de la información, de la comunicación y de la imagen, junto con el uso de las nuevas tecnologías de la comunicación y de la informática (tipo "internet") por dichos sectores. Está demostrada, por experiencias en todos los continentes, la posibilidad de lograr este objetivo, con pocos recursos, así como la eficacia y la influencia que ejerce la utilización de estos medios tanto al interior como en el entorno de los sectores pobres.

\section{Atención a inmigrantes y desla-} zados en situación de pobreza, un grupo social que ha ido aumentando en los últimos años y que, probablemente, va a seguir aumentando, generando situaciones difíciles al interior de numerosos países, con desplazados de su lugar de origen por causas naturales, como terremotos, sequías, hambrunas, etc. como por violencias políticas, étnicas, terrorismo y 
guerras, y por las permanentes olas migratorias en busca de trabajo en otros países. La mayoría de los desplazados y de los inmigrantes viven, por mucho tiempo, en auténtica pobreza, por lo que es necesario considerar programas especiales tanto de emergencia, para atender las necesidades inmediatas, como estructurales orientados a la reubicación segura y estable, con servicios elementales adecuados, en caso de los desplazados, y, en caso de los inmigrantes, mejorando las legislaciones vigentes al respecto y la igualdad de derechos y obligaciones con los ciudadanos de los países que los reciben. También se deben desarrollar programas en las naciones de origen de los movimientos migratorios, ya sea para mejorar las condiciones de vida en el propio lugar, ya sea informando las condiciones legales y económicas que van a tener que aceptar en caso de emigración a otro país.

\section{Cuidado de la Naturaleza y del} Medio Ambiente: generando conciencia de la importancia del tema. Vigilar que los programas de lucha contra la pobreza, especialmente aquellos relacionados con cultivos alternativos y con la minería, que buscan el desarrollo de unas zonas y poblaciones determinadas, no terminen en un deterioro tal del medio ambiente, que, a largo plazo, anule las expectativas de vida digna en dichos lugares. En tales programas deben participar conjuntamente, los ciudadanos y ciudadanas de la zona, los empresarios, los gobiernos nacionales y locales y los expertos en tecnología ambiental, además de mejorar la, legislación nacional. e internacional para que no haya un abuso en la utilización de los recursos naturales. Aprender de experiencias positivas que se han venido practicando en muchos países, especialmente desarrollados y contribuir a que los países pobres puedan mejorar la utilización de dichos recursos, beneficiando económicamente a su población, pero sin dañar el medio, teniendo en cuenta objetivos de corto, mediano y largo plazo.

\section{Canjear deuda externa por desa-} rrollo y educación: esta es una manera real de aliviar a los países pobres de la carga que representa la deuda externa para sus economías, mas aun, si se ha comprobado que los créditos y prestamos otorgados por los países desarrollados y por los organismos financieros internacionales, no siempre han sido utilizados debidamente ni han servido para el beneficio directo de las grandes mayorías de los países deudores, aumentando las desigualdades y, en muchos casos, los niveles de pobreza. Para ello, se deben crear mecanismos de control de estos acuerdos en los que participe directamente la población que se pretende ayudar y no solo los gobiernos centrales y las entidades financieras y empresariales de los países deudores, con el objetivo de que no se repitan los mismos resultados señalados. Se está generando conciencia, en diferentes partes del mundo, sobre la necesidad de hacer realidad esta propuesta, por lo cual fomentar experiencias concretas que culminan en éxito será muy importante para el futuro de las relaciones económico -financieras entre los países en los próximos años.

Estas y otras propuestas y recomendaciones para la lucha contra la pobreza en el mundo se enmarcan, como lo 
hemos venido señalando en las líneas precedentes, en un marco nacional e internacional que considera tres grandes objetivos mundiales para los próximos años: construir nuevas bases para unas relaciones internacionales justas, avanzar hacia una universalización en base al respeto de las culturas particulares y fortalecer la democracia, la ciudadanía y la vigencia de los derechos humanos en todos los niveles. Nos parece que no se puede separar la erradicación de la pobreza de estos tres grandes objetivos, mejor dicho, en la medida en que dichos programas y acciones contribuyan, también, a alcanzarlos, de forma mas rápida y segura iremos disminuyendo la pobreza en todos nuestros países.

Estamos convencidos de que las soluciones existen, que no solamente hay buena voluntad de parte de miles y miles de personas de toda condición y en todos los países, sino que ya hay avances, experiencias concretas que deben ser analizadas, asimiladas y multiplicadas. Debemos asumir la co- responsabilidad ante las causas de un desarrollo desigual y del aumento de la pobreza y sus consecuencias. Asumir la co - responsabilidad desde tos países desarrollados y desde los países en vías de desarrollo, en el Norte y en el Sur, globalizando la solidaridad, reconociendo que existen condiciones y suficientes recursos humanos y naturales para que todos, sin excepción, en este siglo que recién comienza, vivamos con dignidad, aportando, como ciudadanos y ciudadanas, a la construcción de una sociedad, universalizada y justa, en nuestro único Planeta que es, definitivamente, la casa de todos.

\section{Bibliografía Citada}

Azcueta, Michel (2000), Desarrollo integral de Villa El Salvador. Perú. Municipalidad de Lima.

BID (1999), Donde estamos y como nos vemos: América Latina frente al Siglo XXI. Washington. Banco Interameri cano de Desarrollo.

FMCU (2001), Kit ciudades y medio ambiente. París. Federación Mundial de Ciudades Unidas.

Laboratoie du Future (2001), "Les conspirateurs du Future: Cinq Histoires de notre temps". París.

Medicus Mundi (1999), Comunicación Norte-Sur. Pamplona, España.

PNUD (2000). Superar la pobreza humana. Nueva York. Naciones Unidas.

World Bank (2000), Our Dream: a World Free of Poverty. Washington. 